\title{
DOI: https://doi.org/10.24297/jap.v18i.8842
}

\section{Study of Nuclear Structure for ${ }^{36} \mathrm{Si}$ Isotope by Using Shell Model with Several Interactions}

\author{
Ali K. Hasan ${ }^{1}$ and Wafaa A. Almudhafar ${ }^{2}$ \\ 1,2 Department of Physics, Faculty of Education for Girls, Kufa University, Najaf, Iraq \\ 1alikh.alsinayyid@uokufa.edu.iq_2walmudhaffar75@gmail.com
}

\begin{abstract}
In this study, the nuclear shell model was applied to calculate the energy levels and reduced electric quadruple transition probability $\mathrm{B}(\mathrm{E} 2)$ for ${ }^{36} \mathrm{Si}$ isotope using the OXBASH code within $(1 \mathrm{~d} 3 / 2,2 \mathrm{~s} 1 / 2,2 \mathrm{p} 3 / 2,1 \mathrm{f} 7 / 2)$ model space and using (HASN, ZBM2 and VPTH) interactions, As this isotope contains eight neutrons outside ${ }^{28} \mathrm{Si}$ core in the region and when comparing the results of this study with the values. Available process compatibility was acceptable. There was good agreement at level $2^{+}{ }_{1}$, and angular momentum and parity were confirmed for levels $4+, 6+$, and for all interactions, and the value of $B(E 2)$ corresponds well with the only practical value available for the transition $B\left(E 2 ; 2_{1}^{+} \rightarrow 0_{1}^{+}\right)$.
\end{abstract}

Keywords: Shell model, OXBASH Code, ${ }^{36} \mathrm{Si}$ isotope, Energy levels, B(E2).

\section{Introduction}

The nuclear shell model proposed by Mayer and Jensen (Mayer \& Jensen, 1955). This model the basic framework for nuclear structure calculations in terms of nucleons and which entered into nuclear physics more than sixty years ago (Haxel, Jensen, \& Suess, 1949; Mayer, 1949). In this model, where the nucleons can move independently from one another, and where the protons and the neutrons submit obey the Pauli principle, the energy levels and configurations are obtained in an analogous way to that for complex atoms in the Hartree approximation.

The nuclear shell model is based on the analogous model for the orbital structure of atomic electrons in atoms Each nucleon moves Independent from the other, but they are all moving in the nuclear field resulting from them all. This model assumes that the nucleus as a system of A-independent fermions, interacting with each other by means of many body nucleon -nucleon strong forces. The shell model describes nuclear spectra at low energies and transition probabilities rather accurately (Al-Jelawy, 2015).

When all the protons or neutrons in a nucleus are in filled shells, the number of protons or neutrons is called a "magic number". The magic numbers are 2, 8, 20, 28, 50, 82, and 126 . This approach, very successfully, explains the ground state properties of nuclei (S Mohammadi et al., 2015).

Calculations for this isotope were performed in the HASP space-shell model. There were multiple efforts to find perfect empirical Hamiltonians. By 1979 Hasper (Shafeghat et al., 2017) had got empirical Hamiltonians for the lower-part of the HASP-shell. In OXBASH for HASP space model have been proposed 4 different interactions were included (Brown, 2001; Brown et al., 2004). In the present work, The calculation on the description of energy levels of Silicon isotope ${ }^{36} \mathrm{Si}$ have been carried out using the code OXBASH for windows (Brown et al., 2004) in (1d3/2, 2s1/2, 2p3/2, 1f7/2) model space and using the HASN, ZBM2 and VPTH interactions (Bhattacharjee et al., 2016; Shafeghat et al., 2017).

\section{Theory}

The basic rules in the calculations of the shell model is a group of single-particle energies (SPES) and matrix elements of the interaction between two particles of neocons (TBME). This group is called the effective interaction or the Hamilton model space. This Hamiltonian model space can be described in two ways: the first method is "veritable", which is created for a given shell-model space from known data on the free nucleonnucleon force, the second method is "experimental", which is dependent on parameters whose values are determined by stipulating the agreement between measured level energies and shell-model eigenvalues 
(Abed, 2019). In this model protons and neutrons move all active single particle orbits with three constraints, Isospin, Angular momentum and Parity, (Mohammadi \& Sirjani, 2015; Hasan \& Zayed, 2019).

As is well known, the interaction between two protons or two neutrons or proton and neutron is almost the same, $\boldsymbol{v}_{\boldsymbol{\pi} \pi}=\mathbf{v}_{\mathbf{v v}}=\mathbf{v}_{\boldsymbol{\pi v}}$, so Isospin (T) was inserted as a new quantum number.

Single-particle wave functions of a neutron and a proton can be expressed as with the $t=1 / 2$ spinner. The nucleus of $N$ neutrons and $Z$ protons $(A=N+Z)$ can be described by a new quantitative number $T$ and $T_{Z}$ (Lawson \& Lawson, 1980; Mohammadi \& Sirjani, 2015).

$$
T_{Z}=1 / 2(N-Z), 1 / 2(N-Z) \leq T \leq A / 2
$$

For the calculations, the shell model Hamiltonian can be written as (Abed, 2019),

$$
\mathrm{H}=\sum_{i} \epsilon_{i} n_{i}+\sum_{i j k l} V_{i j k l} a_{i}^{\dagger} a_{j}^{\dagger} a_{l} a_{k}
$$

Where $\epsilon_{i}$ : is the single-particle energies (SPE) that can be obtained from neighbors of closed shell nuclei having mass $\mathrm{A}=$ closed core +1 .

$n_{i}$ : the number of nucleons occupying the orbit $\mathrm{i}$.

$V_{i j k l}$ : is the Two-Body Matrix Element (TBME) coupled to good spin and isospin.

$a_{i}^{\dagger} a_{j}^{\dagger}$ : Creation operators (create pair of fermions).

$a_{l} a_{k}$ : Annihilation operators (annihilate pair of fermions).

We can calculate the potential angular momentum states that arise when there is more than one neocon outside the closed core (Lawson \& Lawson, 1980).

First theorem: If there are two similar neocons and they are in the same orbit of the single block the angular momentum is calculated:

$$
J=0,2,4, \ldots, 2 J-1
$$

Second theorem: If similar neoclonin is found at two different levels, one at the level $J_{1}$ and the other at $J_{2}$ where $I_{2} \neq J_{1}$ angular momentum is:

$$
J=J_{1}+J_{2} J_{1}+J_{2}-1, \ldots,\left|J_{1}-J_{2}\right|
$$

Third theorem: In general for similar neocons, if all are in the same orbit, where $n>2$, where $n$ is the number of particles in the outside of the closed shell. the highest value of the angular momentum of $J_{M}$ is (Lawson \& Lawson, 1980):

$$
J_{M}=n\left[j-\frac{(n-1)}{2}\right]
$$

The reduced electric transition probability can be determined by (Brussaard, Glaudemans, \& Klein, 1978):

$$
\mathrm{B}\left(\bar{\omega} L ; J_{i} \rightarrow J_{f}\right)=\frac{\left\langle\mathrm{Jf}\|\mathrm{o}(\bar{\omega} L)\| \mathrm{J}_{\mathrm{i}}\right\rangle^{2}}{2 J_{i}+1}
$$

Where $\left(J_{i}\right.$ and $\left.J_{f}\right)$ are the spin of the initial and final states, respectively and $(\mathrm{O}(\bar{\omega} L))$ is the electric multiple operators. 


\section{Shell model calculation}

The study and calculate the energy levels and probability of the $\mathrm{B}(\mathrm{E} 2)$ transitions using the harmonic oscillator potential $(\mathrm{HO}, b), b<0$ for ${ }^{36} \mathrm{Si}$ isotope. The calculations were performed in the space model $(1 \mathrm{~d} 3 / 2,2 \mathrm{~s} 1 / 2$, $2 \mathrm{p} 3 / 2$ and $1 \mathrm{f7} / 2$ ) above the $N=22$ and $Z=14$ closed shells for neutrons and protons within HASP space by using the OXBASH program (Brown et al., 2004) which has the ability to calculate the equations by executing certain orders after selecting both the effective interaction and the model space The counterpart specified in the study. Based on the values of the single gravity energies and the model space of the studied nuclei as well as the values of the matrix elements using both the HASN, ZBM2 and VPTH interactions, we obtain the energy level values of the ground state, which were compared with the experimental values available to us.

\subsection{Energy Levels}

We can compare the theoretical values of energy levels relative to the ground state of the ${ }^{36} \mathrm{Si}$ nucleus by using HASN, ZBM2, and VPTH interactions with available experimental results for Silicone isotopes shown in the table (1), From this table, we noticed that there is compatibility with the experimental data and according to the total angular momentum values and parity. it shows the following in the all interactions:

1. The angular momentum and parity are identical to the ground state of level $0+{ }_{1}$ when compared with the available experimental data.

2. The agreement is good for the state $2^{+} 1$ as compared with the experimental data.

3. The overall angular momentum and parity of the practical energies $(2.850,3.692)$ were confirmed the practically uncertain and corresponding to the angular momentum $4+, 6+$.

4. We observed that the highest value of theoretically calculated energy in reaction HASN is (18.324)MeV, in reaction ZBM2 is (19.142) MeV and in reaction VPTH is (23.879) MeV, while the highest practically calculated value of energy is (3.692) MeV, and one hundred and eighteen levels were obtained With total angular momentum and parity for all reactions, no practical value for angular momentum, parity and energy was matched in the practical results.

Table 1: Comparing theoretical results of energy levels for $\left({ }^{36} \mathrm{Si}\right)$ nucleus with experimental results (Nica et al., 2012) using (HASN, ZBM2 and VPTH) interactions.

\begin{tabular}{|c|l|l|l|l|l|l|l|}
\hline \multicolumn{9}{|c|}{ Theoretical values Ex (MeV) } & \multicolumn{2}{l|}{ Experimental values } \\
\hline $\boldsymbol{I}^{\boldsymbol{\pi}}$ & HASN & $\boldsymbol{J}^{\boldsymbol{\pi}}$ & ZBM2 & $\boldsymbol{J}^{\boldsymbol{\pi}}$ & VPTH & $\boldsymbol{I}^{\boldsymbol{\pi}}$ & Ex (MeV) \\
\hline $0^{+}{ }_{1}$ & 0.000 & $0^{+}{ }_{1}$ & 0.000 & $0^{+}{ }_{1}$ & 0.000 & $0^{+}$ & 0.000 \\
\hline $2^{+}{ }_{1}$ & 1.906 & $2^{+}{ }_{1}$ & 1.878 & $2^{+}{ }_{1}$ & 2.008 & $2^{+}$ & 1.408 \\
\hline $4^{+}{ }_{1}$ & 2.982 & $4^{+}{ }_{1}$ & 3.060 & $4^{+}{ }_{1}$ & 2.889 & $\left(4^{+}\right)$ & 2.850 \\
\hline $6^{+}{ }_{1}$ & 3.272 & $6^{+}{ }_{1}$ & 3.488 & $6^{+}{ }_{1}$ & 3.114 & $\left(6^{+}\right)$ & 3.692 \\
\hline $2^{+}{ }_{2}$ & 3.487 & $0^{+}{ }_{2}$ & 3.586 & $2^{+}{ }_{2}$ & 4.096 & --- & ------- \\
\hline $4^{+}{ }_{2}$ & 3.578 & $2^{+}{ }_{2}$ & 3.737 & $0^{+}{ }_{2}$ & 4.366 & --- & ------ \\
\hline $3^{+}{ }_{1}$ & 3.701 & $2^{+}{ }_{3}$ & 4.121 & $4^{+}{ }_{2}$ & 4.576 & --- & ------- \\
\hline $5^{+}{ }_{1}$ & 3.712 & $4^{+}{ }_{2}$ & 4.356 & $3^{+}{ }_{1}$ & 4.907 & --- & ------- \\
\hline $0^{+}{ }_{2}$ & 3.807 & $3^{+}{ }_{1}$ & 4.428 & $5^{+}{ }_{1}$ & 4.908 & --- & ------- \\
\hline $0^{+}{ }_{3}$ & 3.951 & $4^{+}{ }_{3}$ & 4.446 & $0^{+}{ }_{3}$ & 5.899 & --- & ------- \\
\hline $2^{+}{ }_{3}$ & 3.988 & $0^{+}{ }_{3}$ & 4.512 & $2^{+}{ }_{3}$ & 5.948 & --- & ------- \\
\hline $2^{+}{ }_{4}$ & 4.663 & $5^{+}{ }_{1}$ & 4.725 & $2^{+}{ }_{4}$ & 6.316 & --- & ------- \\
\hline
\end{tabular}




\begin{tabular}{|c|c|c|c|c|c|c|c|}
\hline $2^{+}{ }_{5}$ & 5.399 & $2^{+}{ }_{4}$ & 4.868 & $2+5$ & 6.427 & --- & ------- \\
\hline $2{ }^{+}{ }_{6}$ & 5.685 & $0^{+}{ }_{4}$ & 5.054 & $4^{+}{ }_{3}$ & 7.136 & --- & ------- \\
\hline $4^{+} 3$ & 5.881 & $2^{+}{ }_{5}$ & 5.100 & $4^{+}{ }_{4}$ & 7.477 & --- & ------ \\
\hline $2^{+} 7$ & 6.180 & $4^{+}{ }_{4}$ & 5.287 & $6^{+} 2$ & 7.478 & --- & ------- \\
\hline $3^{+}{ }_{2}$ & 6.212 & $2^{+} 6$ & 5.448 & $2^{+} 6$ & 7.510 & --- & ------ \\
\hline $0^{+}{ }_{4}$ & 6.232 & $3^{+}{ }_{2}$ & 5.493 & $2^{+} 7$ & 7.676 & --- & ------- \\
\hline $4^{+}{ }_{4}$ & 6.236 & $1^{+}{ }_{1}$ & 5.586 & $3^{+} 2$ & 7.874 & --- & ------- \\
\hline $1^{+}{ }_{1}$ & 6.242 & $2^{+} 7$ & 5.611 & $4^{+}{ }_{5}$ & 7.892 & --- & ------- \\
\hline $4^{+}{ }_{5}$ & 6.309 & $5^{+} 2$ & 5.612 & $2^{+} 8$ & 7.949 & --- & ------- \\
\hline $6^{+}{ }_{2}$ & 6.543 & $6^{+}{ }_{2}$ & 5.695 & $1^{+}{ }_{1}$ & 7.981 & --- & ------- \\
\hline $4^{+}{ }_{6}$ & 6.701 & $0^{+}{ }_{5}$ & 5.708 & $0^{+}{ }_{4}$ & 8.075 & --- & ------- \\
\hline $0^{+}{ }_{5}$ & 6.713 & $4^{+}{ }_{5}$ & 5.763 & $2^{+} 9$ & 8.145 & --- & ------- \\
\hline $2^{+}{ }_{8}$ & 6.717 & $2^{+} 8$ & 5.902 & $5^{+} 2$ & 8.207 & --- & ------- \\
\hline $1^{+}{ }_{2}$ & 6.722 & $2^{+} 9$ & 6.054 & $1^{+}{ }_{2}$ & 8.282 & --- & ------- \\
\hline $3^{+}{ }_{3}$ & 6.747 & $4^{+}{ }_{6}$ & 6.069 & $4^{+}{ }_{6}$ & 8.340 & --- & ------- \\
\hline $4^{+}{ }_{7}$ & 6.775 & $3^{+} 3$ & 6.085 & $0^{+}{ }_{5}$ & 8.633 & --- & ------- \\
\hline $0^{+}{ }_{6}$ & 6.810 & $6^{+}{ }_{3}$ & 6.174 & $3^{+}{ }_{3}$ & 8.684 & --- & ------- \\
\hline $2^{+} 9$ & 6.815 & $5^{+}{ }_{3}$ & 6.200 & $5^{+}{ }_{3}$ & 8.702 & --- & ------- \\
\hline $5^{+}{ }_{2}$ & 6.836 & $0^{+}{ }_{6}$ & 6.261 & $6^{+}{ }_{3}$ & 8.719 & --- & ------- \\
\hline $2^{+} 10$ & 6.852 & $2^{+} 10$ & 6.316 & $4^{+} 7$ & 8.741 & --- & ------- \\
\hline $1^{+}{ }_{3}$ & 6.855 & $4^{+} 7$ & 6.323 & $3^{+}{ }_{4}$ & 8.820 & --- & ------- \\
\hline $5^{+}{ }_{3}$ & 6.866 & $1^{+}{ }_{2}$ & 6.338 & $4^{+}{ }_{8}$ & 8.833 & --- & ------- \\
\hline $6^{+}{ }_{3}$ & 6.892 & $3^{+}{ }_{4}$ & 6.346 & $5^{+}{ }_{4}$ & 8.906 & --- & ------- \\
\hline $3^{+}{ }_{4}$ & 6.970 & $4^{+} 8$ & 6.403 & $1^{+}{ }_{3}$ & 8.947 & --- & ------- \\
\hline $4^{+}{ }_{8}$ & 7.040 & $4^{+} 9$ & 6.454 & $8^{+}{ }_{1}$ & 8.981 & --- & ------- \\
\hline $3^{+}{ }_{5}$ & 7.075 & $0^{+}{ }_{7}$ & 6.553 & $2^{+} 10$ & 8.986 & --- & ------- \\
\hline $5^{+}{ }_{4}$ & 7.077 & $1^{+}{ }_{3}$ & 6.555 & $6^{+}{ }_{4}$ & 9.036 & --- & ------ \\
\hline $0^{+}{ }_{7}$ & 7.080 & $3^{+}{ }_{5}$ & 6.605 & $7^{+}{ }_{1}$ & 9.069 & --- & ------- \\
\hline $3^{+}{ }_{6}$ & 7.114 & $6^{+}{ }_{4}$ & 6.650 & $3^{+}{ }_{5}$ & 9.120 & --- & ------- \\
\hline $4^{+}{ }_{9}$ & 7.118 & $1^{+}{ }_{4}$ & 6.670 & $5^{+}{ }_{5}$ & 9.126 & --- & ------- \\
\hline $4^{+} 10$ & 7.167 & $4^{+} 10$ & 6.680 & $6^{+}{ }_{5}$ & 9.130 & --- & ------- \\
\hline $1^{+}{ }_{4}$ & 7.257 & $8^{+} 1$ & 6.713 & $4^{+} 9$ & 9.145 & --- & ------- \\
\hline $6^{+}{ }_{4}$ & 7.264 & $0^{+}{ }_{8}$ & 6.721 & $0^{+}{ }_{6}$ & 9.154 & --- & ------- \\
\hline $5^{+}{ }_{5}$ & 7.272 & $3^{+}{ }_{6}$ & 6.835 & $4^{+}{ }_{10}$ & 9.222 & --- & ------- \\
\hline $6^{+}{ }_{5}$ & 7.331 & $1^{+}{ }_{5}$ & 6.859 & $5^{+}{ }_{6}$ & 9.231 & --- & ------- \\
\hline
\end{tabular}




\begin{tabular}{|c|c|c|c|c|c|c|c|}
\hline $3^{+}{ }_{7}$ & 7.357 & $5^{+}{ }_{4}$ & 6.962 & $3^{+}{ }_{6}$ & 9.246 & --- & ------ \\
\hline $7^{+}{ }_{1}$ & 7.383 & $3^{+} 7$ & 6.974 & $1_{4}^{+}$ & 9.259 & --- & ------ \\
\hline $8^{+}{ }_{1}$ & 7.395 & $6^{+}{ }_{5}$ & 7.006 & $8^{+}{ }_{2}$ & 9.296 & --- & ------- \\
\hline $1^{+}{ }_{5}$ & 7.417 & $7^{+}{ }_{1}$ & 7.094 & $0^{+}{ }_{7}$ & 9.369 & --- & ------- \\
\hline $0^{+}{ }_{8}$ & 7.418 & $3^{+} 8$ & 7.126 & $6^{+}{ }_{6}$ & 9.418 & --- & -------- \\
\hline $6^{+}{ }_{6}$ & 7.440 & $3_{9}^{+}$ & 7.135 & $3^{+} 7$ & 9.425 & --- & ------- \\
\hline $5^{+}{ }_{6}$ & 7.506 & $5^{+}{ }_{5}$ & 7.142 & $\mathrm{O}_{8}^{+}$ & 9.441 & --- & ------ \\
\hline $3^{+}{ }_{8}$ & 7.578 & $6^{+}{ }_{6}$ & 7.152 & $3^{+} 8$ & 9.609 & --- & ------ \\
\hline $3^{+}{ }_{9}$ & 7.610 & $6^{+} 7$ & 7.188 & $3^{+} 9$ & 9.647 & --- & -------- \\
\hline $7^{+}{ }_{2}$ & 7.633 & $7^{+} 2$ & 7.239 & $1^{+}{ }_{5}$ & 9.665 & --- & -------- \\
\hline $6^{+}{ }_{7}$ & 7.666 & $5^{+}{ }_{6}$ & 7.252 & $\mathrm{O}_{9}^{+}$ & 9.669 & --- & -------- \\
\hline $1^{+}{ }_{6}$ & 7.696 & $\mathrm{O}^{+}{ }_{9}$ & 7.262 & $0^{+}{ }_{10}$ & 9.699 & --- & ------ \\
\hline $5^{+} 7$ & 7.715 & $7^{+}{ }_{3}$ & 7.273 & $7^{+}{ }_{2}$ & 9.742 & --- & ------- \\
\hline $6^{+}{ }_{8}$ & 7.739 & $1^{+}{ }_{6}$ & 7.290 & $1^{+}{ }_{6}$ & 9.751 & --- & ------- \\
\hline $1^{+}{ }_{7}$ & 7.759 & $0^{+} 10$ & 7.338 & $3^{+} 10$ & 9.804 & --- & ------- \\
\hline $0^{+} 9$ & 7.768 & $3^{+} 10$ & 7.389 & $6^{+}{ }_{7}$ & 9.838 & --- & ------ \\
\hline $3^{+}{ }_{10}$ & 7.838 & $5^{+} 7$ & 7.422 & $6^{+} 8$ & 9.888 & --- & -------- \\
\hline $7^{+}{ }_{3}$ & 7.844 & $6^{+} 8$ & 7.510 & $5^{+} 7$ & 9.897 & --- & ------- \\
\hline $5^{+} 8$ & 7.899 & $6^{+} 9$ & 7.569 & $5^{+} 8$ & 9.977 & --- & ------- \\
\hline $6^{+}{ }_{9}$ & 7.947 & $8^{+}{ }_{2}$ & 7.584 & $5^{+} 9$ & 10.090 & --- & ------ \\
\hline $5^{+}{ }_{9}$ & 7.974 & $5^{+} 8$ & 7.595 & $1^{+}{ }_{7}$ & 10.114 & --- & -------- \\
\hline $6^{+}{ }_{10}$ & 8.000 & $5^{+} 9$ & 7.648 & $7^{+}{ }_{3}$ & 10.230 & --- & -------- \\
\hline $5^{+}{ }_{10}$ & 8.021 & $1^{+}{ }_{7}$ & 7.661 & $5^{+}{ }_{10}$ & 10.270 & --- & ------ \\
\hline $7^{+}{ }_{4}$ & 8.021 & $6^{+} 10$ & 7.704 & $1^{+} 8$ & 10.306 & --- & ------- \\
\hline $1^{+} 8$ & 8.049 & $5^{+} 10$ & 7.708 & $6^{+} 9$ & 10.369 & --- & -------- \\
\hline $0^{+}{ }_{10}$ & 8.069 & $7^{+}{ }_{4}$ & 7.764 & $7^{+}{ }_{4}$ & 10.396 & --- & -------- \\
\hline $7^{+{ }_{5}}$ & 8.110 & $8^{+}{ }_{3}$ & 7.949 & $1^{+} 9$ & 10.400 & --- & ------- \\
\hline $1^{+}{ }_{9}$ & 8.143 & $1^{+} 8$ & 7.961 & $6^{+} 10$ & 10.435 & --- & ------- \\
\hline $1^{+}{ }_{10}$ & 8.164 & $1^{+} 9$ & 8.067 & $1^{+}{ }_{10}$ & 10.653 & --- & -------- \\
\hline $8^{+}{ }_{2}$ & 8.166 & $1^{+} 10$ & 8.126 & $7^{+}{ }_{5}$ & 10.692 & --- & -------- \\
\hline $8^{+}{ }_{3}$ & 8.274 & $7^{+}{ }_{5}$ & 8.244 & $10^{+}{ }_{1}$ & 10.753 & --- & ------ \\
\hline $7^{+}{ }_{6}$ & 8.464 & $10^{+}{ }_{1}$ & 8.327 & $7^{+} 6$ & 10.757 & --- & ------ \\
\hline $7^{+}{ }_{7}$ & 8.578 & $8^{+}{ }_{4}$ & 8.380 & $8^{+}{ }_{3}$ & 10.765 & --- & ------- \\
\hline $7^{+}{ }_{8}$ & 8.692 & $7^{+}{ }_{6}$ & 8.430 & $9^{+}{ }_{1}$ & 10.866 & --- & ------- \\
\hline $9^{+}{ }_{1}$ & 8.696 & $8^{+}{ }_{5}$ & 8.632 & $8^{+}{ }_{4}$ & 10.911 & --- & -------- \\
\hline
\end{tabular}




\begin{tabular}{|c|c|c|c|c|c|c|c|}
\hline $8^{+}{ }_{4}$ & 8.751 & $7^{+} 7$ & 8.703 & $8^{+}{ }_{5}$ & 10.928 & --- & ------- \\
\hline $8^{+}{ }_{5}$ & 8.765 & $9^{+}{ }_{1}$ & 8.745 & $7^{+} 7$ & 10.961 & --- & ------ \\
\hline $7^{+} 9$ & 8.866 & $7^{+} 8$ & 8.795 & $7^{+} 8$ & 10.993 & --- & ------ \\
\hline $8^{+}{ }_{6}$ & 8.895 & $7^{+} 9$ & 8.881 & $8^{+}{ }_{6}$ & 11.288 & --- & ------- \\
\hline $10^{+}{ }_{1}$ & 8.927 & $8^{+}{ }_{6}$ & 8.890 & $7^{+} 9$ & 11.315 & --- & ------- \\
\hline $9^{+}{ }_{2}$ & 9.016 & $9^{+}{ }_{2}$ & 8.907 & $9^{+}{ }_{2}$ & 11.481 & --- & ------- \\
\hline $8^{+} 7$ & 9.077 & $7^{+}{ }_{10}$ & 9.061 & $7^{+}{ }_{10}$ & 11.564 & --- & ------ \\
\hline $7^{+}{ }_{10}$ & 9.135 & $9^{+}{ }_{3}$ & 9.150 & $9^{+} 3$ & 11.742 & --- & ------- \\
\hline $9^{+}{ }_{3}$ & 9.160 & $8^{+} 7$ & 9.313 & $8^{+} 7$ & 11.833 & --- & ------- \\
\hline $8^{+} 8$ & 9.203 & $8^{+} 8$ & 9.449 & $8^{+}{ }_{8}$ & 11.891 & --- & ------- \\
\hline $8^{+} 9$ & 9.336 & $9^{+}{ }_{4}$ & 9.515 & $9^{+}{ }_{4}$ & 12.210 & --- & ------- \\
\hline $9^{+}{ }_{4}$ & 9.356 & $8^{+} 9$ & 9.537 & $8^{+} 9$ & 12.248 & --- & ------ \\
\hline $8^{+} 10$ & 9.482 & $9^{+}{ }_{5}$ & 9.601 & $9^{+}{ }_{5}$ & 12.509 & --- & ------- \\
\hline $10^{+}{ }_{2}$ & 9.534 & $8^{+}{ }_{10}$ & 9.683 & $8^{+}{ }_{10}$ & 12.526 & --- & ------- \\
\hline $11^{+}{ }_{1}$ & 9.595 & $10^{+}{ }_{2}$ & 9.715 & $10^{+}{ }_{2}$ & 12.599 & --- & ------- \\
\hline $9^{+}{ }_{5}$ & 9.773 & $10^{+} 3$ & 10.279 & $10^{+}{ }_{3}$ & 12.715 & --- & ------- \\
\hline $9^{+}{ }_{6}$ & 9.888 & $11^{+}{ }_{1}$ & 10.315 & $9^{+}{ }_{6}$ & 12.854 & --- & ------- \\
\hline $10^{+}{ }_{3}$ & 10.011 & $9^{+}{ }_{6}$ & 10.561 & $11^{+}{ }_{1}$ & 12.937 & --- & ------- \\
\hline $9^{+}{ }_{7}$ & 10.018 & $9^{+} 7$ & 10.731 & $10^{+}{ }_{4}$ & 13.053 & --- & ------- \\
\hline $10^{+}{ }_{4}$ & 10.107 & $9^{+}{ }_{8}$ & 11.008 & $9^{+} 7$ & 13.071 & --- & ------- \\
\hline $9^{+}{ }_{8}$ & 10.313 & $9^{+}{ }_{9}$ & 11.287 & $9^{+}{ }_{8}$ & 13.138 & --- & ------- \\
\hline $10^{+}{ }_{5}$ & 10.323 & $10^{+}{ }_{4}$ & 11.292 & $9^{+} 9$ & 13.561 & --- & ------- \\
\hline $9^{+}{ }_{9}$ & 10.473 & $10^{+}{ }_{5}$ & 11.462 & $9^{+}{ }_{10}$ & 14.046 & --- & ------- \\
\hline $9^{+} 10$ & 10.483 & $9^{+} 10$ & 11.580 & $10^{+}{ }_{5}$ & 14.577 & --- & ------- \\
\hline $10^{+}{ }_{6}$ & 10.752 & $10^{+} 6$ & 11.773 & $10^{+}{ }_{6}$ & 14.625 & --- & ------- \\
\hline $10^{+}{ }_{7}$ & 11.437 & $10^{+}{ }_{7}$ & 12.399 & $10^{+}{ }_{7}$ & 14.684 & --- & '------ \\
\hline $11^{+}{ }_{2}$ & 11.638 & $11^{+}{ }_{2}$ & 12.860 & $11^{+}{ }_{2}$ & 14.824 & --- & ------- \\
\hline $10^{+}{ }_{8}$ & 12.035 & $10^{+} 8$ & 13.485 & $10^{+}{ }_{8}$ & 15.291 & --- & ------- \\
\hline $10^{+} 9$ & 12.167 & $10^{+}{ }_{9}$ & 13.809 & $10^{+}{ }_{9}$ & 16.519 & --- & ------- \\
\hline $10^{+}{ }_{10}$ & 14.866 & $10^{+} 10$ & 13.895 & $10^{+} 10$ & 18.199 & --- & ------- \\
\hline $11^{+}{ }_{3}$ & 16.102 & $11^{+}{ }_{3}$ & 13.915 & $11^{+}{ }_{3}$ & 18.867 & --- & ------- \\
\hline $11^{+}{ }_{4}$ & 16.219 & $11^{+}{ }_{4}$ & 15.009 & $11^{+}{ }_{4}$ & 20.402 & --- & ------ \\
\hline $11^{+}{ }_{5}$ & 16.525 & $11^{+}{ }_{5}$ & 15.959 & $11^{+}{ }_{5}$ & 20.821 & --- & ------- \\
\hline $11^{+}{ }_{6}$ & 16.965 & $11^{+}{ }_{6}$ & 16.420 & $11^{+}{ }_{6}$ & 21.474 & --- & ------- \\
\hline $11^{+} 7$ & 17.285 & $11^{+} 7$ & 17.180 & $12^{+} 1$ & 21.670 & --- & ------- \\
\hline
\end{tabular}




\begin{tabular}{|l|l|l|l|l|l|l|l|}
$111^{+}$ & 17.675 & $12^{+}{ }_{1}$ & 17.192 & $11^{+}{ }_{7}$ & 22.224 & --- & ------ \\
\hline $12^{+}{ }_{1}$ & 17.683 & $12^{+}{ }_{2}$ & 18.128 & $11^{+}{ }_{8}$ & 22.394 & --- & ------ \\
\hline $12^{+}{ }_{2}$ & 17.913 & $11^{+}{ }_{8}$ & 18.147 & $11^{+}{ }_{9}$ & 22.534 & --- & ------ \\
\hline $11^{+}{ }_{9}$ & 18.031 & $11^{+}{ }_{9}$ & 18.494 & $12^{+}{ }_{2}$ & 23.569 & --- & ------ \\
\hline $11^{+}{ }_{10}$ & 18.324 & $11^{+}{ }_{10}$ & 19.142 & $11^{+} 10$ & 23.879 & --- & ------ \\
\hline
\end{tabular}

\subsection{Reduced electric quadruple transition probability B(E2) Calculation:}

The probability of reduced electric quadruple transition for $36 \mathrm{Si}$ nucleus in the shell model by using the HASN, ZBM2 and VPTH interactions with the harmonic oscillator (HO). By comparing in Table 2 between the theoretical results and the available practical results until this study was conducted, we found a good agreement for the value of transition ( $\mathrm{B}(\mathrm{E} 2) \mathrm{2}^{+}{ }_{1} \rightarrow \mathrm{O}^{+}{ }_{1}$ ) while there were two transfers that did not have a practical value, the primary polarization effect was included by selecting the effective charge for neutron and protons $(\mathrm{ep}=\mathrm{en}=0.95 \mathrm{e}$ ), respectively for all reactions as shown in Table 2 .

Table 2: Comparison of the theoretical results of $\mathrm{B}(\mathrm{E} 2)$ for $\left({ }^{36} \mathrm{Si}\right)$ nucleus with the experimental data (Nica et al., 2012).

\begin{tabular}{|c|c|c|c|c|}
\hline \multirow[t]{2}{*}{$J_{i}^{\pi} \longrightarrow J_{f}^{\pi}$} & \multicolumn{3}{|c|}{$\begin{array}{r}\text { Theoretical B(E2), } \mathrm{e}^{2} \mathrm{fm}^{4} \\
\mathrm{e}_{\mathrm{p}}=\mathrm{e}_{\mathrm{n}}=0.95 \mathrm{e}\end{array}$} & \multirow[t]{2}{*}{ Experimental $B(E 2), e^{2} \mathrm{fm}^{4}$} \\
\hline & HASN & ZBM2 & VPTH & \\
\hline $2^{+}{ }_{1} \longrightarrow 0^{+}{ }_{1}$ & 37.940 & 38.990 & 31.010 & 38.835 \\
\hline $4^{+}{ }_{1} \longrightarrow 2^{+}{ }_{1}$ & 30.800 & 21.660 & 26.720 & ------- \\
\hline $6^{+}{ }_{1} \longrightarrow 4^{+}{ }_{1}$ & 21.920 & 15.000 & 14.320 & ------ \\
\hline
\end{tabular}

\section{Conclusions}

It is clear from the results obtained in this study:

1- We have noticed the correspondence of the total angular momentum and the parity of the ground state to level 0 for all interactions between the theoretical and practical results available, and there was an acceptable match for the rest of the theoretical energy levels when compared with the practical results.

2- We found that the shell model gives more energy levels with parity and total angular momentum than is practically calculated, and we noticed that the theoretically calculated maximum value for energy is greater than the highest practically calculated value.

3- We noticed a good correspondence in the value of $B(E 2)$ between the theoretical value and the practical value available for this isotope.

4- We conclude from the above that the nuclear shell model is a successful model for studying the nucleus chosen in the study and using the code. 


\section{REFERENCES}

1. Abed, H. H. (2019). Applying Nuclear Shell Model to Study Nuclear Structure for $18 \mathrm{Ne}$ by Using OXBASH Code. Journal of Kufa-physics, 11(02), 1-5.

2. Al-Jelawy, S. S. (2013). No-Core Shell Model Calculations for some Light Nuclei. M.Sc. Thesis, University of Babylon, Babylon, Iraq, 76.

3. Bhattacharjee, R., Das, S., Samanta, S., Bhattacharjee, S. S., Raut, R., Ghugre, S. S., Sinha, A. K., Garg, U., Mukhopadhyay, S., Chakrabarti, R., Dhal, A., Madhavan, N., Singh, R. P., \& Muralithar, S. (2016). High Spin States in 41Ca. Paper presented at the Proceedings of the DAE-BRNS Symp. on Nucl. Phys.

4. Brown, B. A. (2001). The nuclear shell model towards the drip lines. Progress in Particle and Nuclear Physics, 47(2), 517-599.

5. Brown, B. A., Etchegoyen, A., Godwin, N. S., Rae, W. D. M., Richter, W. A., Ormand, W. E., Warburton, E. K., Winfield, J. S., Zhao, L., \& Zimmerman, C. H. (2004). Oxbash for windows PC. MSU-NSCL Report, 1289.

6. Brussaard, P., Glaudemans, P., \& Klein, A. (1978). Shell-Model Applications in Nuclear Spectroscopy. PhT, 31(11), 68.

7. Hasan, A. A., \& Zayed, B. (2019). Study of Nuclear Structure of $18 \mathrm{~F}$ Isotope by using PW and CWH Interactions. Modern Applied Science, 13(12), 52.

8. Haxel, O., Jensen, J. H. D., \& Suess, H. E. (1949). On the" magic numbers" in nuclear structure. Physical Review, 75(11), 1766.

9. Lawson, R., \& Lawson, R. (1980). Theory of the nuclear shell model (Vol. 9): Clarendon Press Oxford.

10. Mayer, M. G. (1949). On closed shells in nuclei. II. Physical Review, 75(12), 1969.

11. Mayer, M. G., \& Jensen, J. H. D. (1955). Elementary theory of nuclear shell structure.

12. Mohammadi, S., Rostami, S. H., Mohasel, A. R., \& Ghamary, M. (2015). Energy Levels Calculations of 36-37-38Ar Isotopes Using Shell Model Code OXBASH. American Journal of Modern Physics. Special Issue: Many Particle Simulations, 4(3-1), 23-26.

13. Mohammadi, S., \& Sirjani, S. (2015). Energy Levels Calculations of 29S, 30 S and 31S Isotopes. American Journal of Modern Physics. Speciallssue: Many Particle Simulations, 4(3-1), 5-9.

14. Nica, N., Cameron, J., \& Singh, B. (2012). Nuclear data sheets for $A=36$. Nuclear Data Sheets, 113(1), 1 155.

15. Shafeghat, H., Teimur, M., \& Mohammadi, S. (2017). Analyzing Energy Levels and Gamma Decay Spectrum Phosphorus-32(14d) Isotope Using OXBASH Shell Model Code for Lower HASP Model Space. Modern Chemistry, 5(5), 82-85. 